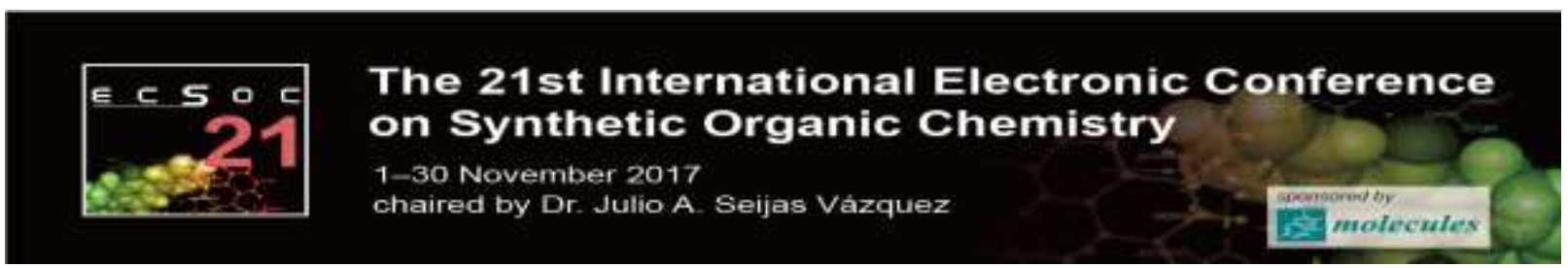

http://sciforum.net/conference/ecsoc-21

\title{
A convenient synthesis of bis(indolyl)methane derivatives using heteroaromatic isocyanurate ring functionalized MCM-41 as a new and highly efficient reusable catalyst
}

\author{
Zahra Alirezvani, Mohammad G. Dekamin*
}
Pharmaceutical and Biologically-Active Compounds Research Laboratory, Department of Chemistry, Iran University of Science and Technology, Tehran 16846-13114, Iran
Tel: +98-21-77240640-50; fax: +98-21-73021584; e-mail: mdekamin@iust.ac.ir

\begin{abstract}
An efficient synthesis of bis(indolyl) methanes (BIM's) has been developed using heteroaromatic isocyanurate ring functionalized MCM-41(HI-MCM-41) as a new nanocatalyst. Purification of products without tedious workup, ease of monitoring and shorter reaction time are the salient features of the developed protocol. The reuse and regeneration of the catalyst have been achieved up to 7 times, respectively, without significant loss of activity.
\end{abstract}

\section{Introduction}

Indole frameworks or scaffold are the most heterocyclic compounds in many biological systems that show medicinal properties and pharmacological activity.[1] During the past, a large numeral of natural products containing bis(indolyl)methanes (BIM's), which contain two indole or substituted indole units in a molecule, have been isolated from marine sources. The BIM's 
exhibit antibacterial, antibiotics, analgesic and anti-inflammatory activities. They are also highly beneficial in promoting good estrogen and increase the body's natural metabolism of hormones.[2, 3]
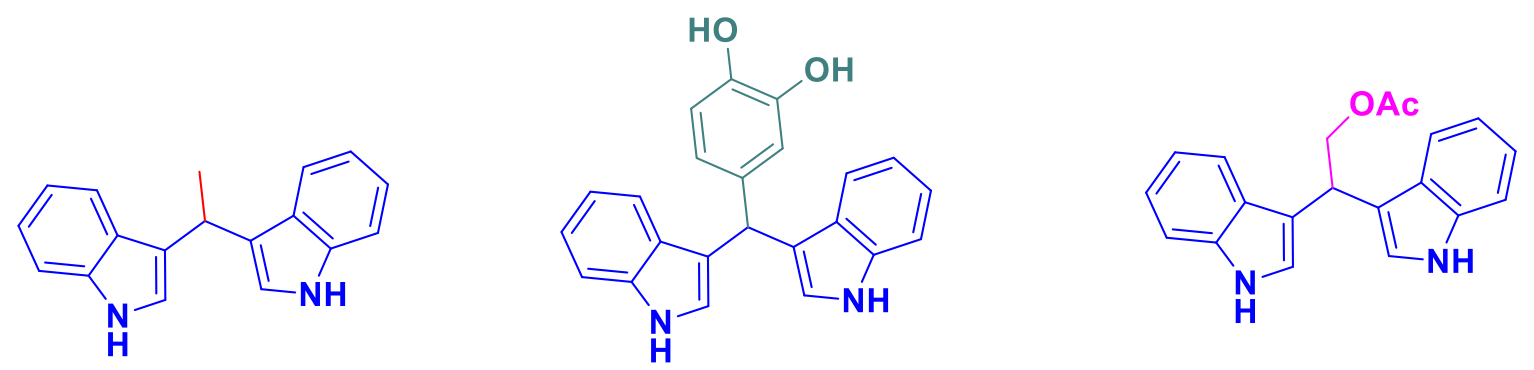

Scheme 1. Representative examples of biologically active bisindolylmethane (BIM) derivatives.

Owing to their many applications, various procedures using different catalyst systems, such as lanthanide triflates, $\mathrm{InCl}_{3}, \mathrm{SBA}-15$-supported poly(4- styrenesulfonyl (perfluorobutylsulfonyl )imide), $\mathrm{NaHSO}_{4} . \mathrm{SiO}_{2} /$ Amberlyst-15 and sulfamic acid are described for the synthesis of bis(indolyl)methanes. However, these methods suffer from certain disadvantages, such as the use of expensive or toxic catalyst, long reaction time, tedious work-up procedures, difficulties in regeneration of catalyst and product purification.[4]

On the other hand, Solid acidic materials have the potential to replace strong liquid acids and preventing corrosion problems associated with them and consequent environmental hazards. Solid acids have found extensive applications in chemical procedures because of their significant advantages including environmental compatibility, non-toxicity, reusability, low cost, high 
activity, operational simplicity and easiness of isolation over strong liquid acids. In continuation of our efforts in the synthesis and application of new heterogenous catalyst for organic transformations[5-8], Herein, we report our results for a facile and convenient synthesis of bis(indolyl)methanes using heteroaromatic isocyanurate ring functionalized MCM-41(HI-MCM41) as an efficient and recoverable catalyst.

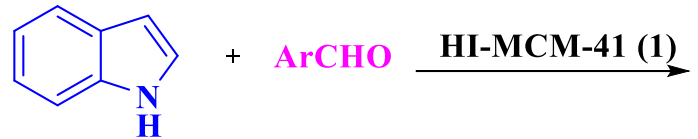

(2)

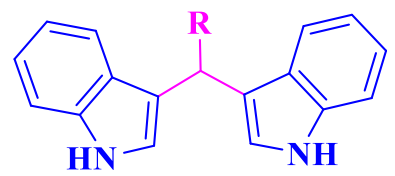

(4)

Scheme 2. Synthesis of bis(indolyl)methane (BIM) derivatives catalyzed by HI-MCM-41

\section{Experimental section}

\section{Reagents and Apparatus}

High-purity chemical reagents were purchased from Merck. All reactions and the purity of the products were monitored by thin-layer chromatography (TLC) using aluminum plates coated with silica gel F254 plates (Merck) using ethyl acetate and n-hexane as eluents. The spots were detected either under UV light or by placing in an iodine chamber. Melting points were determined in open capillaries using an Electrothermal 9100.

General procedure for the synthesis of bis(indolyl)methane derivatives (4a-f)

In a round-bottomed flask, indole $(2,2 \mathrm{mmol})$, various aldehyde $(3,1 \mathrm{mmol})$, and $10 \mathrm{mg} \mathrm{HI}-$ MCM-41 (1) were added to EtOH 96\% (2 mL). The obtained mixture was stirred under reflux conditions. After completion of the reaction monitored by TLC (eluent: $25 \% \mathrm{v} / \mathrm{v}$ EtOAc/n- 
hexane), $\mathrm{EtOH} 96 \%$ (3 mL) was added and the obtained mixture was heated and filtered off to separate the solid catalyst 1 . Water was added dropwise to the filtrate at $50{ }^{\circ} \mathrm{C}$ to give pure crystals of the desired products $\mathbf{4 a - f}$ with yields based on the starting aldehyde.

\section{Results and discussion}

The catalytic facility of the HI-MCM-41 was evaluated in catalyzing a reaction for the efficient synthesis of bis(indolyl)methane derivatives by condensing indole, various aldehydes in refluxing EtOH. The results were evaluated qualitatively through TLC. It was found that the quantitative yield can be achieved when the reaction was carried out in the presence of $0.01 \mathrm{~g}$ catalyst in refluxing EtOH. The results are summarized in Scheme 3. The HI-MCM-41 (1) were easily separated with filtrations and the recovered catalyst was reused for at least 7 runs without significant degradation in catalytic activity and performance (Fig. 1).
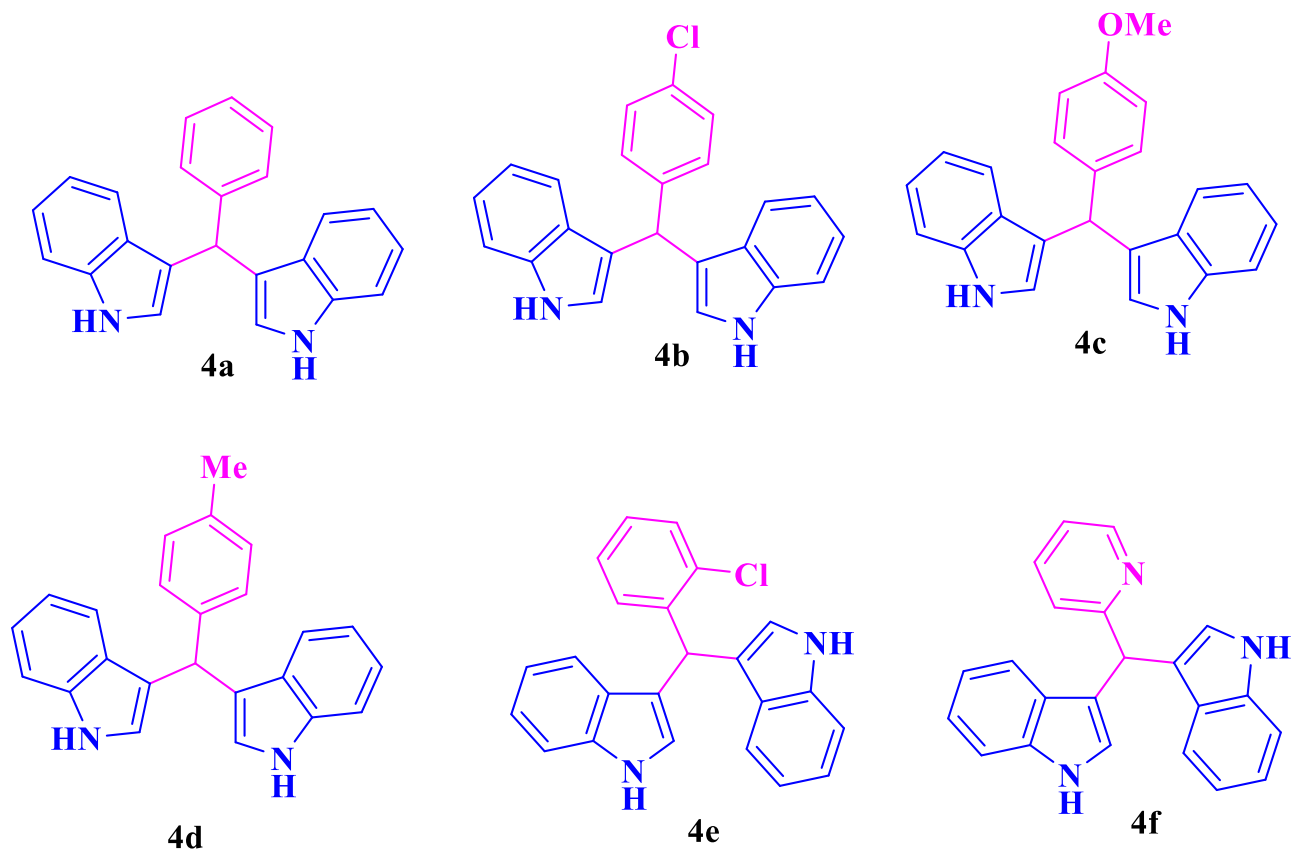

Scheme 3. Scope of bis(indolyl)methane (BIM) derivatives (4a-f) 


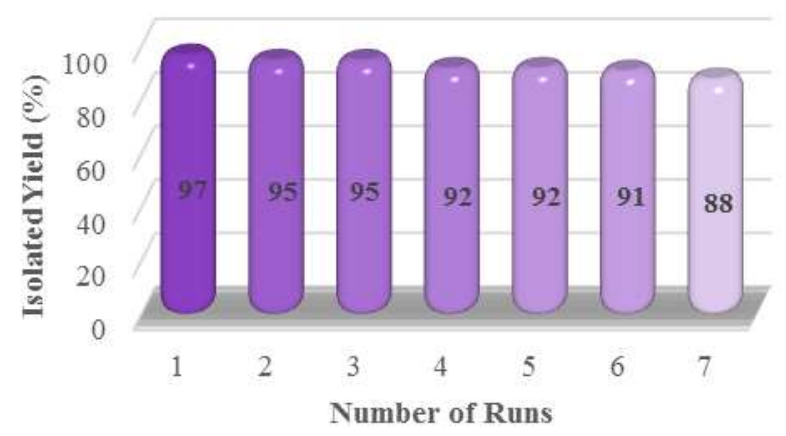

Fig 1. Reusability of HI-MCM-41 (1) nanocatalyst for the synthesis of bis(indolyl)methane derivatives

\section{Conclusion}

We have found that heteroaromatic isocyanurate ring functionalized MCM-41 (HI-MCM-41) can be efficiently utilized for the efficient synthesis of bis(indolyl)methanes. The bis(indolyl)methanes were obtained in good to excellent yield. There are several advantages using this approach, specifically shorter reaction and sequestration duration, low dosage, and reusability. The catalyst can be regenerated and reused without significant loss of activity.

\section{Acknowledgements}

We are grateful for the financial support from The Research Council of Iran University of Science and Technology (IUST), Tehran, Iran (Grant no. 160/8034).

\section{References}


[1] M. Shiri, Indoles in multicomponent processes (MCPs), Chemical reviews 112(6) (2012) 3508-3549.

[2] S. Sarva, J.S. Harinath, S.P. Sthanikam, S. Ethiraj, M. Vaithiyalingam, S.R. Cirandur, Synthesis, antibacterial and anti-inflammatory activity of bis (indolyl) methanes, Chinese Chemical Letters 27(1) (2016) 16-20.

[3] A. Yaghoubi, M.G. Dekamin, E. Arefi, B. Karimi, Propylsulfonic acid-anchored isocyanurate-based periodic mesoporous organosilica (PMO-ICS-Pr-SO3H): A new and highly efficient recoverable nanoporous catalyst for the one-pot synthesis of bis (indolyl) methane derivatives, Journal of Colloid and Interface Science 505 (2017) 956-963.

[4] S. Choudhary, K. Pandey, S. Budania, A. Kumar, Functionalized ionic liquid-assisted chromatography-free synthesis of bis (indolyl) methanes, Molecular diversity 21(1) (2017) 155162.

[5] A. Azad, M.G. Dekamin, S. Afshar, A. Tadjarodi, A. Mollahosseini, Activation of hexamethyldisilazane (HMDS) by TiO2 nanoparticles for protection of alcohols and phenols: the effect of the catalyst phase on catalytic activity, Research on Chemical Intermediates 1-13.

[6] M.G. Dekamin, F. Mehdipoor, A. Yaghoubi, 1, 3, 5-Tris (2-hydroxyethyl) isocyanurate functionalized graphene oxide: a novel and efficient nanocatalyst for the one-pot synthesis of 3 , 4-dihydropyrimidin-2 (1H)-ones, New Journal of Chemistry (2017).

[7] A. Yaghoubi, M.G. Dekamin, B. Karimi, Propylsulfonic Acid-Anchored Isocyanurate-Based Periodic Mesoporous Organosilica (PMO-ICS-PrSO3H): A Highly Efficient and Recoverable Nanoporous Catalyst for the One-Pot Synthesis of Substituted Polyhydroquinolines, Catalysis Letters (2017) 1-8. 
[8] M.G. Dekamin, S. Ilkhanizadeh, Z. Latifidoost, H. Daemi, Z. Karimi, M. Barikani, Alginic acid: a highly efficient renewable and heterogeneous biopolymeric catalyst for one-pot synthesis of the Hantzsch 1, 4-dihydropyridines, RSC Advances 4(100) (2014) 56658-56664. 